\title{
Effect and efficacy of thermal environment provided by a new bathing style, "mist sauna bathing"
}

\author{
Satoshi IWASE ${ }^{1)}$, Yuko KAWAHARA ${ }^{2)}$, Chihiro MIWA ${ }^{3)}$, Naoki NISHIMURA ${ }^{1)}$
}

1) Department of Physiology, School of Medicine, Aichi Medical University, Nagakute 480-1195, Japan

2) Developing Group, Product Development Department, Research \& Development Division, Toho Gas Co. Ltd., Nagoya 456-8511, Japan

3) Department of Occupational Therapy, Medical School of Health Science, Nagoya University, Nagoya 461-

\begin{abstract}
Mist sauna is a style of bathing in which hot water vapor is sprayed into a bathroom, establishing an air temperature of $40^{\circ} \mathrm{C}$ with saturated humidity. Bath heater and dryer equipment with mist sauna function was released onto the Japanese market in 2004. After their introduction, various studies investigated the effects of mist sauna bathing, and it has been demonstrated that mist sauna bathing has various effects and efficacies, not only hyperthermic effects but also other effects including on physical appearance. Mist sauna bathing occurs in a bathroom, usually without a toilet, in which the room temperature is approximately $40^{\circ} \mathrm{C}$ with $100 \%$ relative humidity. It has been shown that the mist sauna causes little hemodynamic change, which ensures its safety during bathing. Therefore, we can enjoy safer physiological bathing in a mist sauna than in traditional hot water immersion bathing. In addition, the mist sauna elicits benefits such as improved skin condition, heat acclimation, and autonomic balance. Since mist sauna bathing does not involve immersion of the body in bathtub water, it is less likely to result in an accident during bathing because of the low impact of hemodynamic changes. Recently, mist sauna bathing has drawn attention in the field of nursing care as a bathing style for the hospitalized elderly that can reduce the burden on care-giving personnel during bathing. It is expected that mist sauna will be adopted by homes and various facilities as a useful approach for various purposes, regardless of the user's age or gender.
\end{abstract}

Key Words: mist sauna, bathing, hyperthermic effect, thermoregulatory function, nursing home

\section{Introduction}

It has been accepted that bathing culture in Japan began with sea bathing in ancient times. Bathing as immersion in hot water became popular among aristocratic people in the Nara period (710-794 AD), and prevalent among the general public in the beginning of the Edo period (1602-1867 AD). In contrast, mist sauna bathing, a bathing form not involving immersion in hot water, has been marketed as one of the functions associated with a bathroom heater since 2004. Subsequently, several gas-supplying companies have studied the merits of heating and the cosmetic effects of mist sauna, and gradually a conception of its beneficial effects and efficacy have penetrated to the general public.

Bathing culture in Japan has been accepted from ancient times as a means of body cleaning as well as relaxation. However, considering the prosperity of public baths in the age of Roman Empire, European culture also had accepted public baths as a social arena. During ancient to medieval times, cleanliness and washing by bathing declined due to Christianity. At the end of the 16th century, Japanese public bath had achieved its position of a social arena among the general public. This style of bathing has continued up until now, but accidents during bathing to death increased at the end of 20th century with increasing the percentage of the aged people. Moreover, as the number of the elderly increased in the nursing care facilities, the burden of care-givers became heavy and heavy during bathing since most of the elderly prefer the hot $\left(>42^{\circ} \mathrm{C}\right)$ water immersion bathing. In the light of European traditional vapor bathing, or saunas, or steam baths, the Japanese household equipment companies tried to incorporate these ways of bathing instead of hot water immersion bathing, however, little is known about thermoregulatory responses to these vapor bathing.

Since mist sauna can be seen at several leisure facilities, such as hot spas or "super sento" (public baths with not only the original sento function, but also other leisure facilities 
including hot spa, open-air bath, several kinds of bathing facility, or sauna, as well as restaurants), the definition of mist sauna seems to be somewhat unclear. Nakayama described that mist sauna involves exposure of the human body to sprayed hot water vapor of approximately $60^{\circ} \mathrm{C}$ in a bathroom, so it is a form of hot water bathing involving a special type of hot water shower, and not heated-air bathing (Nakayama, 2008). He explained that mist sauna is a different type of bathing from heated-air or steamed-vapor bathing.

Some people have discussed that mist sauna is not included in the category of "sauna" bathing, which involves a dry sauna with a dry and high-temperature environment of around $100^{\circ} \mathrm{C}$. Dry sauna was invented in countries in which there are few hours of sunlight in winter, such as Finland and Estonia. In such countries, sauna users expose themselves to a high-temperature environment with low humidity, which causes profuse sweating, and then jump into cold water. This procedure improves their thermoregulatory function through rapid vasoconstrictive activity under an extreme cold environment.

We expose ourselves to a wet and lowtemperature atmosphere in a mist sauna, compared with that in a heated-air sauna or steamed-vapor sauna. In terms of the temperature, a mist sauna is likely to be similar to hot water immersion bathing, which might suggest that a mist sauna can be classified into the category of sauna bathing. This argument makes the classification of mist sauna vague, and some people describe a mist sauna as "mist bathing." This lack of clarity may be due to a lack of understanding about the characteristics of a mist sauna.

In the present review, we would like to clarify the characteristics of mist sauna by describing its thermal environment and the associated effects and benefits that have been identified to date. In addition to this, we will describe the possible expansion of mist sauna use into the field of welfare, utilizing a bathing form that does not involve immersion in hot water. In the present review, we use the term "mist sauna" to refer to the environmental conditions, and "mist bathing" as the procedure of bathing in a mist sauna.

\section{Production method of mist sauna}

There are three ways to produce mist: 1) nozzle splash method, 2) vaporization method, and 3) water cracking method (Table 1). In the vaporization method, hot water at a temperature of $60^{\circ} \mathrm{C}$ is dropped on the surface of a heat converter heated by $80^{\circ} \mathrm{C}$ water, blown by a fan, and vaporized to produce humidification of $100 \% \quad \mathrm{RH}$ (relative humidity). In the water cracking method, which has been developed recently, small nozzle-sprayed water particles are exposed to hot air in order to heat them; then, the hot and humid air is sent into the bathroom by a fan. A demerit of this system is the low humidification capability, which is $60-90 \%$ $\mathrm{RH}$ depending on the air tightness of the bathroom. The diameter of the particles in the vaporization and water cracking methods is less than $10 \mu \mathrm{m}$, which is called micro-mist. In the conventional nozzle splash method, hot water is directly splashed from a nozzle. This method is the simplest, and is easily produced in an existing bathroom by bifurcating the water tap. Since the method splashes mist into the air directly, the diameter of the particles is approximately 100 to $200 \mu \mathrm{m}$, which is large enough for users to get wet immediately. Another name for the mist made by this method is "splash mist." Recently, a hybrid system combining the micro-mist and splash mist has been developed, in which the user can employ either method.

What are the differences in the effects of micro-mist and splash mist on the human body? Kawahara and Kuwano (2011) compared the effects of micro-mist produced by the water cracking method and splash mist by the conventional nozzle splash method, and reported that the tympanic temperature (core temperature) and the mean skin temperature increased slower and there was less heat sensation upon micro-mist exposure than with splash mist exposure (Fig. 1). This difference is considered to be due to the difference in the water contained in the bathroom air, since the humidity is not saturated in micro-mist, and little micro-mist is present in the bathroom. These two factors might be the main causes of the more moderate heating effects on the human body 
in the micro-mist than in the splash mist approach.

\section{Basic study on mist sauna}

In an early study on mist sauna, Miwa et al. (1999) investigated the effect of mist heating using a mist sauna facility by the nozzle splash method as a potentially physiologically safe heating method; they reported that mist heating is favorable for elderly people, who are considered to be easily dehydrated, because it causes less sweating than warm-air heating. Sekimoto et al. (2001) surveyed the effect of mist sauna at 38,40 , and $42^{\circ} \mathrm{C}$ splash, and concluded that higher-temperature splash mist caused greater increases in heart rate and body temperature.

In terms of a vaporization mist sauna, Kawahara et al. (2002) compared its effects with those of a dry sauna with a room temperature at $70^{\circ} \mathrm{C}$ and a relative humidity of $10 \%$; they reported that the mist sauna had less effect on the cardiovascular system and resulted in milder dehydration by sweating, indicating its physiologically safe effects, whereas there were higher rates of vasodilatation and sweating per body temperature rise, which showed the efficient thermal effects of a mist sauna (Fig. 2). Nishikawa et al. (2000) compared the thermal effects of head-out bathing involving immersion in $40^{\circ} \mathrm{C}$ hot water for $10 \mathrm{~min}$ and vaporization mist sauna bathing with micromist at $40^{\circ} \mathrm{C}$ and $100 \% \mathrm{RH}$, from the viewpoint that mist bathing is a form of hot water immersion bathing. They showed that, although a systolic pressure drop was observed during head-out hot water immersion bathing, mist sauna bathing provided no fluctuation in systolic blood pressure and a good bathing feeling, both of which were due to the high-humidity conditions $(\sim 100 \% \mathrm{RH})$ of the mist sauna.

A recent study by Kawahara et al. (2005) examined the effect and efficacy of micromist by the water cracking method; they reported that thermal effects were significantly correlated to specific enthalpy, dry- and wet-bulb temperatures, and relative humidity, and that skin-moisturizing action was well correlated to relative humidity. They concluded that this action could not be obtained unless the relative humidity was high, even with a high-temperature environment in the sweating temperature zone (Fig. 3).

These studies indicated that exposure to the environment with a relatively low temperature $\left(\sim 40^{\circ} \mathrm{C}\right)$ and a high relative humidity $(100 \%)$ provided by mist sauna bathing is an innovative way of bathing, which produces physiologically safe and comfortable bathing conditions.

\section{Efficacy of a mist sauna, by applying the temperature and humidity of a mist sauna environment}

Several features resulting from mist sauna bathing have beneficial effects on the human body. Kawahara et al. (2005) investigated the effect of light exercise during mist sauna bathing. Focusing on light exercise as the desired performance during mist sauna bathing in market research, they examined the effects of stretching and resistance training during mist sauna bathing. Subjects were instructed to perform isotonic exercise (arm swinging with a frequency of $1 \mathrm{~Hz}$ ) in a mist sauna environment, which resulted in an increase in serum free fatty acid level and a decrease in blood glucose level without changing the body temperature, indicating the energy metabolism was enhanced by exercise in the mist sauna. Miwa et al. (2010) determined the metabolic rate during exercise in a mist sauna, and disclosed that the metabolic rate during mist sauna bathing with isotonic exercise (arm swinging with a frequency of $1 \mathrm{~Hz}$ ) was twice as great as that during only mist sauna exposure, corresponding to exercise of $12 \mathrm{~min}$ of walking at a speed of $70 \mathrm{~m} / \mathrm{min}$. Han et al. (2006) measured the trapezius muscle blood flow by near-infrared spectrometry during "mist stretch bathing." They also evaluated the refreshing and comfortable feeling in the neck and shoulders using the visual analog scale method. It was found that the combination of stretching exercise and mist sauna bathing increased the trapezius muscle blood flow, and enhanced the refreshing and comfortable feeling by "mist stretch bathing." Concerning the beneficial effect of mist sauna on allergic rhinitis, Hotehama et al. (2006) reported such effect of mist sauna bathing on nasal allergy as a result of the humidified 
environment. They exposed patients with allergic rhinitis to mist sauna bathing and bathtub bathing, had the patients assess the improvement of nasal obstruction by the visual analog scale, and measured the nasal cavity volume by acoustic rhinometry. As a result, they observed that an improvement continued for more than $30 \mathrm{~min}$ after the mist sauna bathing, which was sustained for more than $90 \mathrm{~min}$; they concluded that mist sauna bathing was effective for improving nasal allergy symptoms.

In a study on the thermal effects of mist sauna on blood flow improvement and facilitation, Yamada et al. examined its effects on neck and shoulder stiffness (2008a) and blood flow in the scalp (2008b) compared with those of bathtub bathing. In the neck and shoulder stiffness study, they exposed 12 women aged $30-40$ years old to 1) $40^{\circ} \mathrm{C}$ mist sauna for 10 min, 2) $40^{\circ} \mathrm{C}$ mist sauna with stretch exercise for $10 \mathrm{~min}$, and 3) head-out hot water immersion at $40^{\circ} \mathrm{C}$ in a bathtub for $10 \mathrm{~min}$. Then, they compared the trapezius muscle stiffness just after, $15 \mathrm{~min}$ after, and $30 \mathrm{~min}$ after the exposure. The results showed that muscle stiffness was lowest in the mist and stretch, intermediate in the mist sauna bathing, and highest in the bathtub bathing, indicating that the muscle blood flow was improved in this order. They also reported that the trapezius muscle stiffness upon applying an oil massage was the same as upon undergoing mist sauna bathing. They estimated that the softening of the trapezius muscle was attributable to a muscle temperature rise, and indicated that the free movement during mist sauna bathing contributed to the better improvement of neck and shoulder stiffness by combining the stretch exercise and the mist sauna. As for the scalp blood flow improvement, they examined the effect of mist sauna bathing on the significance of scalp care in male pattern alopecia. They measured the scalp skin temperature with a thermistor and the skin blood flow with a laser Doppler flowmeter, and observed increases in both parameters. Therefore, mist sauna bathing was shown to be beneficial for scalp hair by improving the scalp skin blood flow.
Ishibashi et al. (2011) examined the effects of mist sauna on relieving mental strain as well as physical strain. They compared the effects of 1) head-out bathtub bathing, 2) shower bathing, and 3) mist sauna bathing on the changes in parameters reflecting physical strain, including average frequency of electromyogram and skin blood flow measured by near-infrared spectroscopy, before and after bathing, by contraction loading of the biceps brachii at the level of $30 \%$ of maximal voluntary contraction until completion. The recovery from the physical strain was observed to be the same between bathtub bathing and mist sauna bathing. To assess the mental strain, a word-detecting test, a mental arithmetic test, and an auditory oddball task were applied before and after the bathing; a latency delay of P300, an amplitude decrease in $\mathrm{Cz}$ through the auditory odd-ball task, and changes in flicker value as well as task performance were observed, indicating that the effect of the mist sauna on recovery from mental strain was the same as that with bathtub bathing.

Concerning the body temperature change after mist sauna bathing, Michihiro et al. (2010) proposed using mist sauna bathing during summer by employing tap water without changing the temperature. They investigated the body temperature and sweating changes before and after $40^{\circ} \mathrm{C}$ mist sauna bathing for $7 \mathrm{~min}$, followed by tap water mist sauna bathing for $3 \mathrm{~min}$. They observed the same rise not only in body temperature and sweating but also in skin moisture as in the usual mist sauna bathing for $10 \mathrm{~min}$, whereas sweating was suppressed and hemodynamic changes were minimized after bathing. Yoshida et al. (2010) investigated the effect of mist sauna bathing on deepening of non-REM sleep stage, and observed that the mist sauna bathing did not influence the sleep efficiency, the latency of sleep onset, or the arousal index, but enhanced the delta wave power in the power spectra of an electroencephalogram for nonREM sleep, indicating that the mist sauna bathing caused deepening of the non-REM sleep stage compared with the bathtub bathing. 
The effects of facial cooling combined with bathtub and mist sauna bathing were investigated by Kawahara et al. (2003); they compared the effects of bathtub bathing in $38^{\circ} \mathrm{C}$ water up to the epigastrium and headout hot water bathtub bathing of $42^{\circ} \mathrm{C}$ for 20 min. They added head-out mist sauna bathing at $42^{\circ} \mathrm{C}$ and $100 \%$ relative humidity for 20 min with and without facial cooling to the bathtub bathing. They found that the tympanic temperature rise was suppressed to $1 / 6$ to $1 / 7$, and the esophageal temperature was also suppressed to $1 / 10$ by bathtub bathing in $38^{\circ} \mathrm{C}$ water up to the epigastrium with facial cooling, compared with those upon bathtub bathing at $42^{\circ} \mathrm{C}$, and that facial cooling provided thermal comfort and postbathing refreshment.

These observations indicate that mist sauna bathing with light exercise facilitates an increase in energy metabolism, and with stretch exercise increases the blood flow of skeletal muscle by thermal and massage stimulation, providing refreshment. It also has relaxing effects on muscle stiffness by enhancing the skeletal muscle blood flow as well as recovery effects on mental strain by attenuating the sympathetic excitation of skeletal muscles. Moreover, the highertemperature and higher-humidity environment of a mist sauna provides several beneficial health effects, including for nasal allergy, neck and shoulder stiffness, enhancement of scalp blood flow, and deep sleep. In order to prevent too great an increase in brain temperature, local cooling, such as facial fanning, might be a good approach to suppress the core temperature.

\section{Effect of serial exposure to mist sauna; chronic effects of mist sauna}

The studies described above mainly focused on the effects and efficacy of a mist sauna upon a single exposure, namely, acute effects. However, mist sauna has many benefits for individuals upon repeated exposure (chronic effects). Here, the efficacies of serial mist sauna bathing are described.

Murakami et al. (2005) examined the cosmetic effects of serial mist sauna bathing for a month on the female skin, and observed a significant increase in water content in the facial stratum corneum and a significant reduction in the facial transepidermal water loss, signifying the improvement of skin surface morphology and the refinement of the facial skin texture. They concluded that mist sauna bathing enhances the penetration of skin care products after bathing, and adjusts the skin condition to strengthen the effects of the products.

As for the improvement of a chilly feeling in the extremities in women, Nakahara et al. (2007) investigated the effect of serial mist sauna bathing for one month on mitigation of such a feeling in the hands and feet of women with oversensitivity to cold. They measured not only the skin temperature and the skin blood flow but also subjective reports of a chilly feeling in their hands and feet; they observed significantly higher skin temperature and significantly higher skin blood flow. The subjects reported a warm sensation in their fingers, which mitigated the chilly sensation in their toes. They also observed suppression of reduced water content in the skin after serial mist sauna bathing, which is usually reduced in winter. Subjective assessment of the skin condition revealed that their skin conditions, namely, skin moisturizing, were improved after serial mist sauna bathing for a month.

Katsumata et al. (2009) verified whether the chilly sensation in their fingers and toes was mitigated by serial mist sauna bathing. They examined the recovery time after immersing their feet in cold water, and observed a significantly shorter recovery time. They also found that "Fatigue" score in The Profile of Mood States was significantly decreased after serial mist sauna bathing. They estimated that serial mist sauna bathing for a certain period is effective for recovery of the autonomic dysfunction caused by thermal stress associated with the movement between rooms with different temperatures, such as summer lethargy, represented by "air-conditioner sickness."

Shiobara (2009) examined the cosmetic effect of serial mist sauna bathing for two weeks. She observed that serial exposure to mist sauna bathing significantly improved skin elasticity, the release rate of skin after suction, and skin color brightness at the cheek area of the subjects, which indicates that the 
incorporation of mist sauna bathing with halfbody bathing (bathing involving immersion in water at a mild temperature of $\sim 38^{\circ} \mathrm{C}$ up to the level of the epigastrium) provides significant improvement of skin condition.

Mist sauna bathing has also been proved to be effective in ameliorating metabolic syndrome in men. Michihiro et al. (2009) surveyed the effect of serial mist sauna bathing for 3 months on the indices of metabolic syndrome in middle-aged men. They measured 1) body fat percentage, 2) body weight, and 3) abdominal circumference, before and after 3 months of everyday exposure to serial mist sauna bathing, and observed significant decreases in these parameters by $1.02 \%, 1.5$ $\mathrm{kg}$, and $2.84 \mathrm{~cm}$, respectively.

The heat acclimation effect of serial mist sauna bathing was examined by Takemori et al. (2009). They determined the sweat volume and the sodium concentration in sweat after $15 \mathrm{~min}$ of $50 \mathrm{~W}$ ergometric exercises before, during (at 1.5 months), and after 3 months of serial mist sauna bathing in middle-aged men. Sweat sodium level was significantly reduced after exposure for 1.5 months, and was further reduced by $65.7 \%$ after 3 months of serial exposure, whereas sweat volume was significantly increased by $89.6 \%$ after 3 months of exposure. These changes indicated the heat acclimation function associated with an increase in the sweat volume along with a decrease in the sodium excretion in the sweat caused by facilitation of the sodium reabsorption in the sweat gland. In another review (2009) on mist sauna bathing, Takemori described that these serial exposure effects are brought about in combination with the thermal effect and skin-moisturizing effect of mist sauna bathing.

These studies of serial mist sauna bathing demonstrated that serial exposure provides cosmetic effects for the skin, enhances the effects of skin care products, and facilitates the recovery of autonomic dysfunction caused by the thermal stress associated with the temperature difference. Furthermore, the possibility that serial exposure to mist sauna for 3 months had an effect on metabolic syndrome was suggested, and a possible preventive effect on heat stroke was indicated by its facilitation of heat acclimation through effective sweating.

\section{Standardization of mist sauna bathing}

Research and development departments of urban gas companies, which have studied the effectiveness and efficacy of mist sauna bathing, proposed standardization of the temperature and time for bathing. The proposed room temperature might be $40^{\circ} \mathrm{C}$, and the recommended time for exposure would be $10 \mathrm{~min}$ in consideration of physiological safety. There is no standardization for relative humidity since mist sauna bathing uses a room with abundant small water particles in the air, which saturates the water vapor in the room at $100 \%$. This high humidity of $100 \%$ renders the dissipating effect of sweating invalid, which might cause dehydration by profuse sweating depending on the room temperature and bathing duration. However, since mist sauna bathing by the water cracking method is incapable of increasing the relative humidity to $100 \%$, the thermal effects in a bathing room with this approach at $40^{\circ} \mathrm{C}$ are different from those with other methods, enabling the evaporating effect of sweat to lower the core temperature effectively (Kawahara and Kuwano, 2011). A bathroom temperature of $40^{\circ} \mathrm{C}$ with a relative humidity of $100 \%$ provides the following changes, according to previous reports, and also depending on seasonal differences: increase in the core temperature by $0.45-0.66^{\circ} \mathrm{C}$ (tympanic), $0.6-0.9^{\circ} \mathrm{C}$ (sublingual), and 0.23 $0.27^{\circ} \mathrm{C}$ (rectal), and increase in the heart rate by 15-20 bpm (Table 2, Kawahara et al. 2002, Kawahara et al. 2005, Sekimoto et al. 2001, Takemori 2009, Nishikawa and Murakami 2000, Han and Takemori 2006, Huang et al. 2009, Maekawa 2008, Michihiro et al. 2010, Miwa and Kawahara 2010, Miwa et al. 2009). These values approximate the changes in the core temperature (tympanic temperature) of a rise of $0.5^{\circ} \mathrm{C}$ and the heart rate elevation of 20 bpm as suggested by Kawahara et al. (2004) when they proposed the physiologically safe and psychologically comfortable changes in the core temperature and the heart rate during bathtub bathing up to the neck. From these observations, it is reasonable to set the 
standardized room temperature to $40^{\circ} \mathrm{C}$ and the mist sauna bathing duration to $10 \mathrm{~min}$. Since this standard is based on the exposure to mist sauna produced by the nozzle splash method and the vaporization method, application of this standard to mist sauna bathing by the water cracking method might provide insufficient thermal comfort to bathers since the mist sauna produced by this method produces mild physiological alteration in the bathers. Different standards are expected to be proposed for mist sauna bathing produced by the water cracking method.

\section{Studies on mist sauna bathing for the children}

In spite of favorable results from the adult men and women, children were reported to be unfavorable preference, and mothers claimed the difficulty in taking a mist sauna with children. Kawahara et al. (2012) examined and determined the best thermal environment of mist sauna for children, employing the mist sauna facility with the changeable direction and angle for mist spraying by the nozzle splashing method. As the result, they showed that the spray nozzle should be directed not to the bathtub but to the washing area, which induced significant thermal effects on children in the washing area. This specific spraying has been approved as preferable heating by children.

Since family bathing is preferred by Japanese families, this result provided a reasonable explanation to account for prevalence of family mist bathing.

\section{Studies on mist sauna bathing for the elderly}

According to a survey by The Tokyo Metropolitan Medical Examiner's Office in 2000 (Tokyo Metropolitan Emergency Association, 2000), sudden death during bathing occurred predominantly in winter, and annually, 14,000 people were estimated to have died in association with bathing, including deaths by disease and accidents, in Japan. Furthermore, cases involving elderly people aged 65 years and older constituted $87.3 \%$ of domestic deaths by accident or by drowning in association with bathing. The fact that elderly people make up a large proportion of those dying during bathing might be due to their insensitivity to hot or cold temperature. The elderly may not feel so strong a stress upon a change in their environment, which might in turn convey a strong physical strain.

Bathing is the specific condition through which elderly people would receive this strong stress. However, there are four specific dangerous situations for elderly people who undertake Japanese-style bathing in winter (Tochihara 2006). The first is when they are exposed to a cold environment in the dressing room. This cold stress elevates their blood pressure. The second is when they enter a hot bathtub. This action would elevate their blood pressure further. The third is when they immerse themselves in hot water in the bathtub, which gradually dilates the blood vessels to lower the blood pressure. This enhances the risk of cerebral or myocardial infarction in conjunction with the dehydration produced by sweating. The fourth is when they leave the bathtub and cease immersion in hot water. Hot water immersion dilates the vessels and lowers the vascular compliance in the lower body. When they stand up to leave the bathtub, massive fluid shift to the lower body occurs due to gravity and reduces the cerebral blood flow, which might be a cause of orthostatic hypotension. These large fluctuations in blood pressure in association with bathing would not occur during mist sauna bathing. It is thus possible to reduce deaths during bathing when elderly people adopt mist sauna bathing instead of conventional bathing immersed in hot water.

As an appropriate method for bathing by the elderly, Maekawa (2008) proposed mist sauna use because it precludes the possibility of drowning accidents. He examined the product of heart rate and systolic blood pressure (the double product) during bathing, and reported that the changes in this double product during mist sauna bathing were significantly smaller than those during bathtub bathing, suggesting that the cardiac load during mist sauna bathing was smaller than that during bathtub bathing, and mist sauna bathing seems to be safer for the elderly. He also assessed mist sauna bathing in terms of subjective thermal sensation, comfort, fatigue sensation, and relaxing sensation using 7-point Likert scales, 
and reported the same satisfaction with mist sauna bathing as with bathtub bathing. Furthermore, he evaluated mist sauna bathing as a safer and easier bathing method not only for the elderly but also for care-receivers. For three elderly care-receivers who had disabilities in terms of walking but could bathe at home, he compared the posture of the care-receivers using a posture discriminator during mist sauna bathing and bathtub bathing. He found that the average anteflexion angle of the care-receivers during mist sauna bathing was smaller than that during bathtub bathing, and the total cumulative time with anteflexion posture at an angle of more than $45^{\circ}$ during mist sauna bathing was shorter than that during bathtub bathing, depending on the condition of the care-receivers and the bathing environment in the care-receivers' home. Therefore, he concluded that mist sauna bathing is a bathing method associated with lower physical strain for elderly care-receivers compared with bathtub bathing.

\section{Study of mist sauna bathing and its possible application to nursing care}

Since mist sauna bathing has been clarified as a low-burden bathing approach for carereceivers, the authors discussed the possible application of a mist sauna in nursing care. Home nursing care has become prevalent for Japanese patients who want to be cared for in their home; however, care-giving tasks by family members are sometimes burdensome, among which bathing care is one of the most burdensome tasks. Because mist sauna bathing does not involve movement over a bathtub edge, the care-receiver does not need to undertake difficult movements in the bathroom. In mist sauna bathing care, the caregiver transfers the care-receiver to the bathroom, after which the care-receiver can stay in the washing area to heat and wash the body. Then, the caregiver simply withdraws the care-receiver from the bathroom, without transfer between the bathtub and the washing area.

Miwa et al. examined the caregiver's burden of caring for simulated hemiparetic patients by comparing the electromyographic (EMG) activity for bathing care between mist sauna and bathtub bathing. The EMG activity for the mist sauna bathing was found to be smaller than that for the bathtub bathing: by $53 \%$ for the shoulder muscles, $43 \%$ for the upper arms, $41 \%$ for the lumbar muscles, and $64 \%$ at the femur. The care time was also shortened to half as long as for bathtub bathing. In addition, POMS showed that the rating of "Fatigue" was significantly reduced by the mist sauna bathing.

Kawahara et al. (2011) reported the merits of introducing a mist sauna facility for home nursing care by interviewing elderly couples. They observed that the introduction of a mist sauna system enabled self-support by the care-receiver and reduced the burden on the caregiver. They concluded that mist sauna bathing contributed to improved quality of life for care-giving family members.

Kashima (2007) discussed the selection of a particular pattern of air flow in a mist sauna facility as a care support system, and proposed several specific flow patterns for caregivers and care-receivers, including changes in the flow direction or cessation of flow during care-giving tasks such as body washing.

The above reports described the beneficial use of a mist sauna facility as a care-giving measure in home nursing care. However, is a mist sauna also beneficial to reduce a caregiver's burden to care for large groups of patients for a long time? Kawahara et al. (2010) tried to answer this by surveying the bathing care in a nursing care home facility affiliated to a hospital. In the surveyed nursing care home facility, bathing care was given in a general public bath and in a bathing facility using a hydraulic elevator for bedridden patients. In the general public bath, caregivers transferred the patients by immersing their lower legs in the bath, and washed the patients' bodies in a humid washing area, which not only forced the caregivers to transfer the patients up and down but also exposed the caregivers to vapor. It was observed that these strains resulted in leukocytosis in the caregivers after bathing care. In the questionnaires from the caregivers after bathing care, there were complaints about working in a hot and humid atmosphere, heavy work intensity, and unbearable posture during care-giving. 
Surveys on subjective symptoms revealed that the caregivers suffered from generalized fatigue, and POMS (Profile on Mood Status) showed that they maintained a negative mood. As for the problems of transferring patients up and down and heat exposure, introduction of machine-based bathing care by using an automatic bathtub or a bathtub with a hydraulic lift may solve the caregivers' problems to some extent; however, this would not be suitable to treat large numbers of bathing patients. Therefore, they concluded that it would be necessary to take countermeasures including the introduction of a mist sauna room specifically for bathing, and to separate the spaces for bathing and for washing to resolve the above work-related problems.

Thus, in terms of the application of a mist sauna to nursing care, it has been suggested that a mist sauna could be beneficial to reduce the burden of caregivers. Almost all the mist sauna instruments are registered as welfare equipment on the webpage of the Technical Aids Information System run by The Association for Technical Aids (http://www.techno-aids.or.jp/system/index.shtml), which can be browsed by specialists associated with generalized welfare, including care managers and welfare caretakers.

\section{Summary}

The present review describes basic studies on the thermal effects conveyed by the thermal environment produced by a mist sauna, as well as applied studies showing the effects and efficacies associated with the thermal effects of a mist sauna. It also describes the application of mist sauna facilities to nursing care, utilizing the merits of a bathing mode that does not involve immersion of the body in water. Mist sauna bathing has been proved beneficial not only for the bathers but also for caregivers to reduce their care burden. It is expected that mist saunas will be adopted by homes and various facilities as a useful tool for various purposes, regardless of the user's age or gender.

\section{Acknowledgments}

The authors would like to thank Ms. Maki Kouroki, Institute for Urban Life, Tokyo Gas Co. Ltd., Ms. Hiroko Fujimura, New Value Development Group, Department of Product
Development, Tokyo Gas Co. Ltd., and Mr. Kazutoshi Takemori, Institute for Energy Technology, Osaka Gas Co. Ltd., for providing research materials on mist saunas.

\section{References}

1. Han JD and Takemori K (2006). New utilization of the mist sauna -Efficacy of mist stretch bathing -. The $30^{\text {th }}$ Symposium on Human-environment System.30: 65-68.

2. Hotehama M, Takemori K, Hiraoka T and Hagino $\mathrm{S}$ (2006). Mist sauna effect on nasal allergy. Perspectives on Otorhinolaryngology 49: 37-40.

3. Huang J, Oda K, Hirasawa Y, Shimomura Y and Katsuura T (2009). The comparison of physiological responses between mist sauna bathing and tub bathing. Journal of Japan Society for Physiological Anthropology 14: 124-125.

4. Ishibashi S, Kouroki M, Soomin L, Shimomura Y and Katsuura T (2011). Comparison of effects of bathing methods on recovery from fatigue. Japanese Journal of Physiological Anthropology 16: 64-65.

5. Kashima M (2007). Bathing support for carereceivers by using the mist sauna. Regional Caring 9: 44-47.

6. Katsumata H, Tsuzuki K, Hirasawa Y (2008). Effects of regularly mist sauna bathing on cold condition in summer. Technical papers of Annual Meeting the Society of Heating, Air-conditioning and Sanitary Engineers of Japan 1267-1270.

7. Kawahara Y, Nagata M, Niimi Y, Miwa C, Iwase S (2002). Effects on changes in hemodynamic and thermoregulatory functions during mist and dry sauna bathing. The Autonomic Nervous System 39: 402-408.

8. Kawahara Y, Iwase S, Miwa C, Kataoka Y, Watanabe Y (2003). Effect of head-out mist bathing and facial fanning on thermoregulatory and cardiovascular functions and thermal comfort during half-body bathing in humans. The Autonomic Nervous System 40:43-50.

9. Kawahara Y, Saito T, Kuno S, Iwase S, Miwa C (2004). Correlation between subjective thermal perception and physiological changes during bathing in winter. Journal of Human and Living Environment 11: 9-19.

10. Kawahara Y, Iwase S, Sugenoya J, Miwa C, Takada M (2005). Effects of mist-sauna bathing on the cardiovascular function, thermoregulatory function, and metabolism of exercising humans. The Autonomic Neruous System 42: 344-351.

11. Kawahara Y, Miwa C, Deguchi A, Mizutani Y (2010). Current state of bathing care and necessity of new equipment for bathing care in nursing care institution. Journal of Human and Living Envrionment 17: 23-30.

12. Kawahara Y, Miwa C Iwase S (2011). The design for the new bathing form, "mist sauna." Symposium for "The assessment and design of various thermal environment," - The expansion of thermal sensation study -. Summary of Technical Papers of Annual Meeting Architectural Institute of Japan21-26. 
13. Kawahara Y, Kuwano Y (2011). Difference of hyperthermia on human during mist sauna bathing between the mist generation method. Summary of technical papers Annual Meeting of Architectural Institute of Japan 41011: 21-22.

14. Kawahara Y, Kuwano Y, Nishimura N, Iwase S (2011). A study on physiological effects of micro mist sauna bathing. Technical Papers of the Annual Meeting of the Society of Heating, Air-conditioning and Sanitary Engineers of Japan. 1869-1872.

15. Kawahara Y, Kuwano Y, Nishimura N and Iwase $\mathrm{S}$. Thermal environment of mist sauna bathing by the nozzle splashing method for children bathers. Technical Papers of the Annual Meeting of the Society of Heating, Air-conditioning and Sanitary Engineers of Japan. 2012: in press

16. Maekawa K (2008). Mist sauna bathing for the elderly. Japan Society of Plumbing Engineers 25: 1216.

17. Michihiro K, Han J, Takemori T (2009). Effect and efficacy of serial mist sauna bathing for three months. Reduction in body weight, body fat percentage, and abdominal circumstance -. Proceedings of the $30^{\text {th }}$ Annual Meeting of Japan Society for the Study of Obesity 15: 227.

18. Michihiro K, Yoshida I, Yamazaki M, Takemori T (2010). A mist sauna bathing style in summer using "water mist". The $34^{\text {th }}$ Symposium on Humanenvironment System 101-104.

19. Miwa C, Iwase S, Mano T (1999). Mist heat effects on thermoregulatory function during bathing at $41^{\circ} \mathrm{C}$ in humans. The Autonomic Nervous System 36: 478-484.

20. Miwa C, Kawahara Y, Yoshida K (2009). Application of mist-sauna on bathing care for the disable. Journal of Human and Living Environment 16: 85-89.

21. Miwa C, Kawahara Y (2010). Effect of exercise on metabolic rate during mist sauna bathing. The $34^{\text {th }}$ Symposium on Human-environment System 263-264.

22. Murakami M, Kawamura A, Hotehama M, Matsunami H, Tanno O (2005). The efficacy of daily mist bathing on physiological functions of skin. Proceedings of the Third International Conference on Human-Environment System 514.
23. Nakahara K, Hirata K, Takemori K (2007). Mitigation effect of the serial mist sauna bathing on the improvement of extremital chilly feeling. Japanese Journal of Biometeorology 44: S32.

24. Nakayama M (2008). Sauna Bathing, this and that. Japan Sauna and Spa Association: Tokyo 138-139.

25. Nishikawa K, Murakami K (2000). Effects of sauna with warm mist on physiological responses. The Japanese Journal of Ergonomics. 36: 316-317.

26. Sekimoto A, Murakami K, Shimomura Y, Iwanaga K Katsuura T (2001). Ergonomic study on mist sauna bathing. Summary of Japanese Journal of Physiological Anthropology 6: 62-63.

27. Shiobara M (2009). Livelihood Front 48. Cosmetic effect of foods and air conditioners. Journal of Home Economics of Japan 60: 181-182.

28. Takemori K (2009). The Seven benefits of mist sauna bathing that originate from condensation at the skin surface. Journal of Heat Transfer Society of Japan 48: $38-43$.

29. Takemori K. Michihiro K, Yamazaki M (2009). Heat acclimation effect by serial exposure to the mist sauna. Japanese Journal of for Biometeorology 48: 3843.

30. Tochihara H (2006). Bathing of Japanese people. Its merits and demerits. Center of Excellence Program for $21^{\text {st }}$ Century Workshop, Safety and Comfortableness of Bathing, School of Design, Faculty of Design, Kyushu University, Fukuoka 2-5.

31. Tokyo Emergency Medical Association (2001). Survey Research Study Report 2000. 27-15.

32. Yamada K, Shimizu T (2008). The effect of mist bathing on the scalp blood. Journal of Japanese Association of Physical Medicine, Balneology and Climatology 71: 167-172.

33. Yamada K Shimizu T (2008). Relaxation effect of mist sauna bathing on shoulder and neck stiffness. Journal of Japanese Association of Physical Medicine, Balneology and Climatology 72: 63.

34. Yoshida I, Takemori K, Yamazaki M, Michihiro K, Tsuzuki K, Urade Y, Yoshida M (2010). Effect of mist sauna bathing on sleep. The $34^{\text {th }}$ Symposium on Human-environment System 34: 106-108 


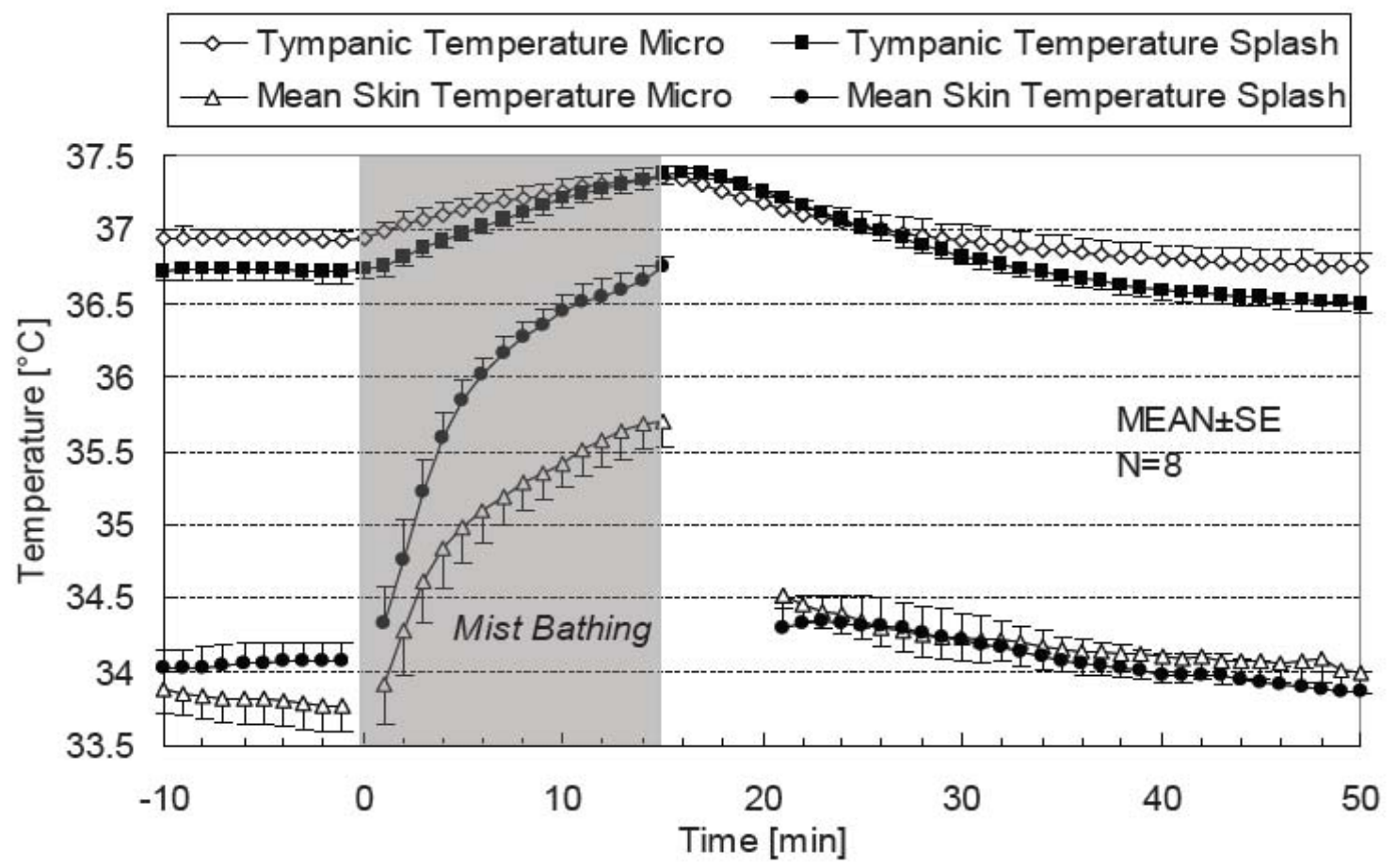

Figure 1. Changes in tympanic temperature (core temperature) and averaged mean skin temperature during exposure to micro-mist and splash mist.

Tympanic temperature (core temperature) and mean skin temperature are shown in the course of mist sauna bathing (micro-mist and splash mist) for $15 \mathrm{~min}$. More moderate increase in tympanic temperature is observed in the mist sauna bathing by micro-mist, depending on the particle diameter (modified from Kawahara and Kuwano, 2011).

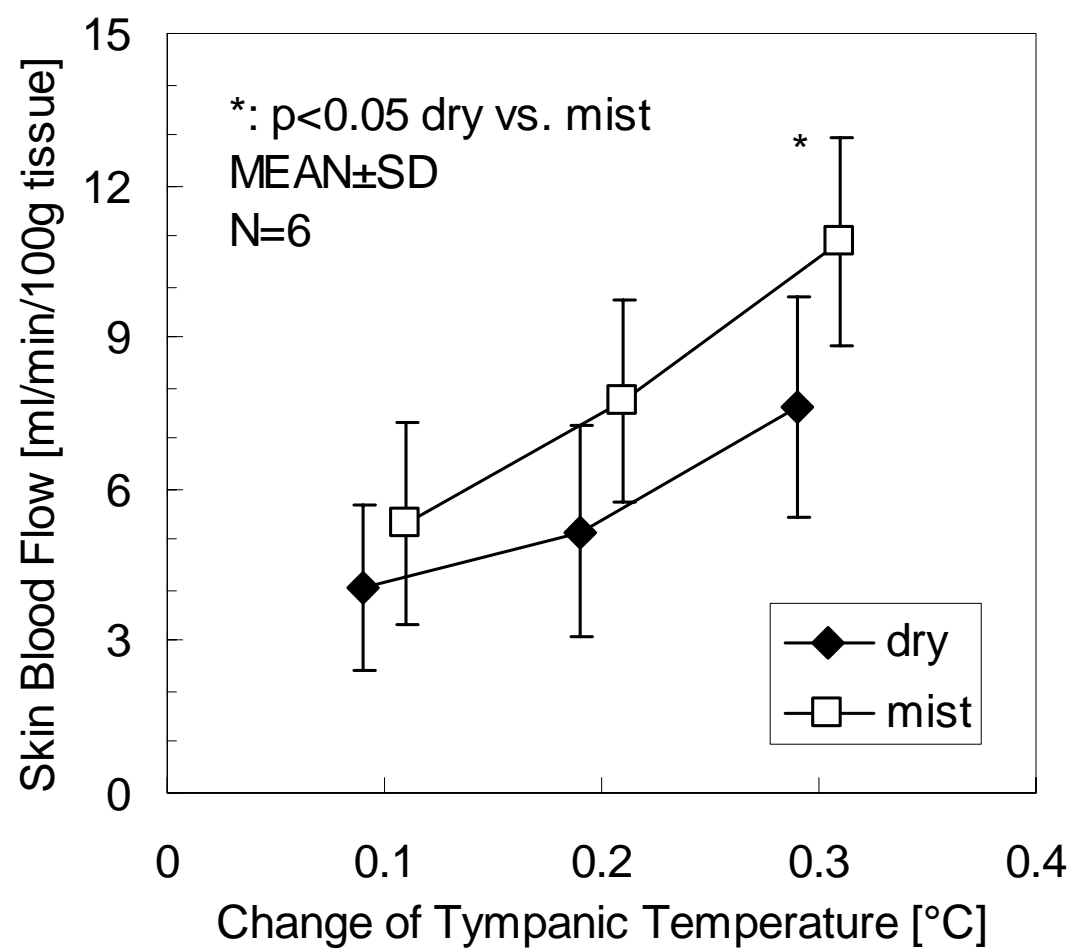

Figure 2. Ratio of the skin blood flow increase to the tympanic temperature (core temperature) rise.

The increase in the forearm skin blood flow measured by laser Doppler flowmetry as a function of the core temperature rise was compared between the dry sauna bathing $(\diamond)$ and the mist sauna bathing $(\square)$. The increase in skin blood flow when the core temperature was raised by $0.3^{\circ} \mathrm{C}$ was significantly larger in the mist sauna bathing than in the dry sauna (modified from Kawahara et al. 2002). 


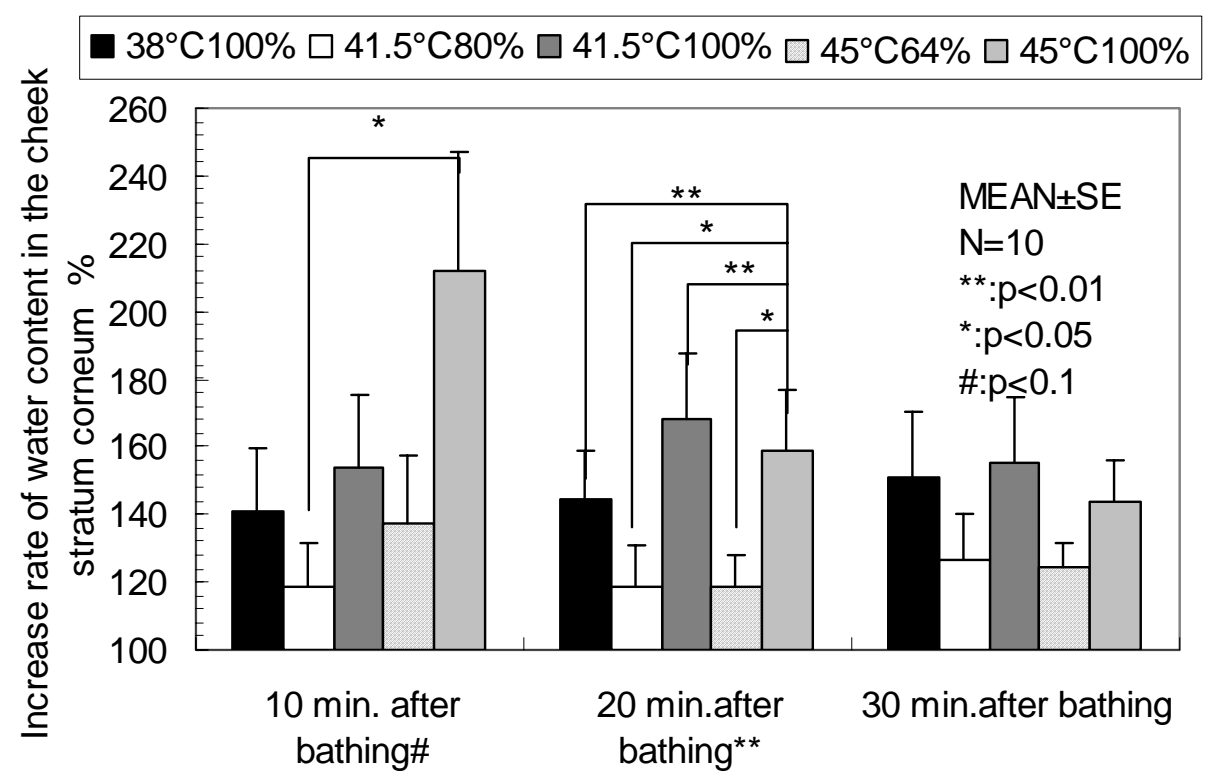

Figure 3. The rate of increase of water content in the cheek stratum corneum in the course of mist sauna bathing by micro-mist.

The rates of increase of water content in the cheek stratum corneum in five conditions: 1) $38^{\circ} \mathrm{C}$ and $100 \% \mathrm{RH}$, 2) $41.5^{\circ} \mathrm{C}$ and $\left.80 \% \mathrm{RH}, 3\right) 41.5^{\circ} \mathrm{C}$ and $\left.100 \% \mathrm{RH}, 4\right) 45^{\circ} \mathrm{C}$ and $64 \% \mathrm{RH}$, and 5) $45^{\circ} \mathrm{C}$ and $100 \% \mathrm{RH}$, at 10,20 , and 30 min after the bathing are shown. \# and * show significant difference among the conditions (\#: $\mathrm{p}<0.1, * *$ : $\mathrm{p}<0.01$ ). The rate of increase was significantly higher in the $100 \% \mathrm{RH}$ conditions irrespective of the bathroom temperature (modified from Kawahara et al. 2011).

Table 1 Mist production method and the mist characteristics

\begin{tabular}{|llll|}
\hline Mist production method & Nozzle splash method & Vaporization method & Water cracking method \\
\hline Mist classification & Splash mist & Micro-mist & Micro-mist \\
\hline Particle diameter & $100-200 \mu \mathrm{m}$ & $1-3 \mu \mathrm{m}$ & $\geq 1 \mu \mathrm{m}$ \\
\hline Relative humidity & $100 \%$ & $100 \%$ & $60-90 \%$ \\
\hline
\end{tabular}

Table 2 Changes in physiological parameters after 10 min of mist sauna bathing

\begin{tabular}{|c|c|c|c|}
\hline $\begin{array}{l}\text { Effects and } \\
\text { efficacy }\end{array}$ & Index & Parameter change & Reference \\
\hline Thermal effect & $\begin{array}{l}\text { Increase in body } \\
\text { temperature }\end{array}$ & $\begin{array}{l}0.45-0.96^{\circ} \mathrm{C} \\
\text { (tympanic) } \\
0.55-0.90^{\circ} \mathrm{C} \\
\text { (sublingual) } \\
0.23-0.27^{\circ} \mathrm{C} \text { (rectal) } \\
\end{array}$ & $\begin{array}{l}\text { Kawahara et al. 2002, Kawahara et al. 2005, } \\
\text { Sekimoto et al. 2001, Takemori 2009, Han et } \\
\text { al. 2006, Huang et al. 2009, Maekawa 2008, } \\
\text { Michihiro et al. 2010, Miwa and Kawahara } \\
\text { 2010, Miwa et al. 2009 }\end{array}$ \\
\hline Sweating & Body weight decrease & $\begin{array}{l}129-210 \mathrm{~g} \text { (winter) } \\
129-261 \mathrm{~g} \text { (summer) }\end{array}$ & $\begin{array}{l}\text { Kawahara et al. 2002, Kawahara et al. 2005, } \\
\text { Sekimoto A et al. 2001, Takemori 2009, } \\
\text { Nishikawa and Murakami 2000, Huang et al. } \\
\text { 2009, Michihiro et al. 2010, Miwa and } \\
\text { Kawahara 2010, Miwa et al. } 2009\end{array}$ \\
\hline $\begin{array}{l}\text { Skin- } \\
\text { moisturizing } \\
\text { effect }\end{array}$ & $\begin{array}{l}\text { Water content in the } \\
\text { stratum corneum of the skin } \\
15-30 \text { min after bathing in } \\
\text { comparison with the pre- } \\
\text { bathing value }\end{array}$ & $\begin{array}{l}1.2 \text { times (elderly) } \\
1.3 \text { times (female) } \\
1.4 \text { times (male) }\end{array}$ & $\begin{array}{l}\text { Takemori 2009, Nishikawa and Murakami } \\
\text { 2000, Maekawa 2008, Michihiro et al. } 2010\end{array}$ \\
\hline $\begin{array}{l}\text { Physiological } \\
\text { safety }\end{array}$ & Heart rate increase & $\begin{array}{l}18-20 \mathrm{bpm} \text { (winter) } \\
15-29 \mathrm{bpm} \text { (summer) }\end{array}$ & $\begin{array}{l}\text { Kawahara et al. 2002, Kawahara et al. 2005, } \\
\text { Sekimoto et al. 2001, Michihiro et al. 2010, } \\
\text { Miwa and Kawahara 2010 }\end{array}$ \\
\hline
\end{tabular}

